九州大学学術情報リポジトリ

Kyushu University Institutional Repository

\title{
Anti-Commutative Dual Complex Numbers and 2D Rigid Transformation
}

Matsuda, Genki

Kyushu University

Kaji, Shizuo

Yamaguchi University | JST CREST

Ochiai, Hiroyuki

Kyushu University | JST CREST

ht tp://hdl. hand le. net/2324/1430820

出版情報 : MI lecture note series. 50, pp.128-133，2013-10-21. 九州大学マス・フォア・インダスト リ研究所

バージョン：

権利関係 : 


\title{
Anti-commutative Dual Complex Numbers and 2D Rigid Transformation
}

\author{
G. Matsuda ${ }^{1}$, S. Kaji ${ }^{2}$, and H. Ochiai ${ }^{3}$
}

Kyushu University ${ }^{13}$, Yamaguchi University ${ }^{2}$, JST/CREST ${ }^{23}$, Japan

The $n$-dimensional rigid transformation (or Euclidean) group $\mathrm{E}(n)$ consists of transformations of $\mathbb{R}^{n}$ which preserves the standard metric. It is well-known (see [2], for example) that any element of $\mathrm{E}(n)$ can be written as a composition of a rotation, a reflection, and a translation, and hence, it is represented by $(n+1) \times(n+1)$-homogeneous matrix;

$$
\mathrm{E}(n)=\left\{A=\left(\begin{array}{cc}
\hat{A} & d_{A} \\
0 & 1
\end{array}\right) \mid \hat{A}^{t} \hat{A}=I_{n}, d_{A} \in \mathbb{R}^{n}\right\} .
$$

Here, we adopt the convention that a matrix acts on a (column) vector by the multiplication from the left. $\mathrm{E}(n)$ has two connected components. The identity component $\mathrm{SE}(n)$ consists of those without reflection. More precisely,

$$
\mathrm{SE}(n)=\left\{A=\left(\begin{array}{cc}
\hat{A} & d_{A} \\
0 & 1
\end{array}\right) \mid \hat{A} \in \mathrm{SO}(n), d_{A} \in \mathbb{R}^{n}\right\},
$$

where $\operatorname{SO}(n)=\left\{R \mid R^{t} R=I_{n}\right.$, $\left.\operatorname{det}(R)=1\right\}$ is the special orthogonal group composed of $n$-dimensional rotations.

The group $\mathrm{SE}(n)$ is widely used in computer graphics such as for expressing motion and attitude, displacement, deformation, skinning, and camera control. In some cases, the matrix representation of the group $\mathrm{SE}(n)$ is not convenient. In particular, a linear combination of two matrices in $\mathrm{SE}(n)$ does not necessarily belong to $\mathrm{SE}(n)$ and it causes the notorious candy-wrapper defect in skinning. When $n=3$, another representation of $\mathrm{SE}(3)$ using the dual quaternion numbers (DQN, for short) is considered in [5] to solve the candy-wrapper defect. In this talk, we consider the 2-dimensional case. Of course, $2 \mathrm{D}$ case can be handled by regarding the plane embedded in $\mathbb{R}^{3}$ and using DQN, but instead, we introduce the anti-commutative dual complex numbers (DCN, for short), which is specific to $2 \mathrm{D}$ with much more concise and faster implementation. We also developed a library for DCN and demonstrate it with an iPad application.

\section{Anti-Commutative dual COmplex numbers}

Let $\mathbb{K}$ denote one of the fields $\mathbb{R}, \mathbb{C}$, or $\mathbb{H}$, where $\mathbb{H}$ is the quaternion numbers. First, we recall the standard construction of the dual numbers over $\mathbb{K}$.

Definition 1. The ring of dual numbers $\hat{\mathbb{K}}$ is the quotient ring defined by

$$
\hat{\mathbb{K}}:=\mathbb{K}[\varepsilon] /\left(\varepsilon^{2}\right)=\left\{p_{0}+p_{1} \varepsilon \mid p_{0}, p_{1} \in \mathbb{K}\right\} .
$$

The addition and the multiplication of two dual numbers are given as

$$
\begin{aligned}
\left(p_{0}+p_{1} \varepsilon\right)+\left(q_{0}+q_{1} \varepsilon\right) & =\left(p_{0}+q_{0}\right)+\left(p_{1}+q_{1}\right) \varepsilon, \\
\left(p_{0}+p_{1} \varepsilon\right)\left(q_{0}+q_{1} \varepsilon\right) & =\left(p_{0} q_{0}\right)+\left(p_{1} q_{0}+p_{0} q_{1}\right) \varepsilon .
\end{aligned}
$$


The following involution is considered to be the dual version of conjugation

$$
\overline{p_{0}+p_{1} \varepsilon}:=\bar{p}_{0}-\bar{p}_{1} \varepsilon,
$$

where $\bar{p}_{i}$ is the usual conjugation. The unit dual numbers are of special importance.

Definition 2. Let $|\hat{p}|=\sqrt{\hat{p} \overline{\hat{p}}}$ for $\hat{p} \in \hat{\mathbb{K}}$. The unit dual numbers is defined as

$$
\hat{\mathbb{K}}_{1}:=\{\hat{p} \in \hat{\mathbb{K}}|| \hat{p} \mid=1\} \subset \hat{\mathbb{K}} .
$$

$\hat{\mathbb{K}}_{1}$ acts on $\hat{\mathbb{K}}$ by conjugation action

$$
\hat{p} \diamond \hat{q}:=\hat{p} \hat{q} \hat{p}
$$

where $\hat{p} \in \hat{\mathbb{K}}_{1}, \hat{q} \in \hat{\mathbb{K}}$.

The unit dual quaternion $\hat{\mathbb{H}}_{1}$ is successfully used for skinning in [5]; a vector $v=$ $(x, y, z) \in \mathbb{R}^{3}$ is identified with $1+(x i+y j+z k) \varepsilon$ and the conjugation action of $\hat{\mathbb{H}}_{1}$ preserves the embedded $\mathbb{R}^{3}$ and its Euclidean metric. In fact, the conjugation action induces the double cover $\hat{\mathbb{H}}_{1} \rightarrow \mathrm{SE}(3)$.

On the other hand, when $k=\mathbb{R}$ or $\mathbb{C}$, the conjugation action is trivial since the corresponding dual numbers are commutative. We define the anti-commutative dual complex numbers $\check{\mathbb{C}}$ by modifying the multiplication of $\hat{\mathbb{C}}$. That is, $\check{\mathbb{C}}=\hat{\mathbb{C}}$ as a set, and the algebraic operations are replaced by

$$
\begin{aligned}
\left(p_{0}+p_{1} \varepsilon\right)\left(q_{0}+q_{1} \varepsilon\right) & =\left(p_{0} q_{0}\right)+\left(p_{1} \bar{q}_{0}+p_{0} q_{1}\right) \varepsilon \\
\overline{p_{0}+p_{1} \varepsilon} & =\bar{p}_{0}+p_{1} \varepsilon \\
\left|p_{0}+p_{1} \varepsilon\right| & =\left|p_{0}\right| .
\end{aligned}
$$

The addition and the conjugation action are left unmodified. Then,

Theorem 3. $\check{\mathbb{C}}$ satisfies distributive and associative laws, and hence, has a (noncommutative) ring structure.

Similarly to the unit dual quaternion numbers, the unit anti-commutative complex numbers are of particular importance:

$$
\check{\mathbb{C}}_{1}:=\{\hat{p} \in \check{\mathbb{C}}|| \hat{p} \mid=1\}=\left\{e^{i \theta}+p_{1} \varepsilon \in \check{\mathbb{C}} \mid \theta \in \mathbb{R}\right\} .
$$

It is a group with inverse

$$
\left(e^{i \theta}+p_{1} \varepsilon\right)^{-1}=e^{-i \theta}-p_{1} \varepsilon
$$

and it acts on $\check{\mathbb{C}}$ by the conjugation action. Now, we regard $\mathbb{C}=\mathbb{R}^{2}$ as usual. Identifying $v \in \mathbb{C}$ with $1+v \varepsilon \in \check{\mathbb{C}}$, we see that $\check{\mathbb{C}}_{1}$ acts on $\mathbb{C}$ as rigid transformation.

\section{Relation to SE(2)}

In the previous section, we constructed the unit anti-commutative dual complex numbers $\breve{C}_{1}$ and its action on $\mathbb{C}=\mathbb{R}^{2}$ as rigid transformation. Recall that $\hat{p}=p_{0}+p_{1} \varepsilon \in$ $\check{\mathbb{C}}_{1}$ acts on $v \in \mathbb{C}$ by

$$
\hat{p} \diamond(1+v \varepsilon)=\left(p_{0}+p_{1} \varepsilon\right)(1+v \varepsilon)\left(\bar{p}_{0}+p_{1} \varepsilon\right)=1+\left(p_{0}^{2} v+2 p_{0} p_{1}\right) \varepsilon,
$$

that is, $v$ maps to $p_{0}^{2} v+2 p_{0} p_{1}$. For example, when $p_{1}=0, v \in \mathbb{C}$ is mapped to $p_{0}^{2} v$, which is the rotation around the origin of degree $2 \arg \left(p_{0}\right)$ since $\left|p_{0}\right|=1$. On the other 
hand, when $p_{0}=1$, the action corresponds to the translation by $2 p_{1}$. In general, we have

$$
\begin{aligned}
& \varphi: \check{\mathbb{C}}_{1} \rightarrow \mathrm{SE}(2) \\
& p_{0}+p_{1} \varepsilon \mapsto\left(\begin{array}{ccc}
\cos \left(2 \arg \left(p_{0}\right)\right) & -\sin \left(2 \arg \left(p_{0}\right)\right) & \operatorname{Re}\left(2 p_{0} p_{1}\right) \\
\sin \left(2 \arg \left(p_{0}\right)\right) & \cos \left(2 \arg \left(p_{0}\right)\right) & \operatorname{Im}\left(2 p_{0} p_{1}\right) \\
0 & 0 & 1
\end{array}\right),
\end{aligned}
$$

where $\operatorname{Re}\left(2 p_{0} p_{1}\right)$ (respectively, $\left.\operatorname{Im}\left(2 p_{0} p_{1}\right)\right)$ is the real (respectively, imaginary) part of $2 p_{0} p_{1} \in \mathbb{C}$. Note that this gives a surjective group homomorphism $\varphi: \check{\mathbb{C}}_{1} \rightarrow \operatorname{SE}(2)$ whose kernel is $\{ \pm 1\}$. That is, for any $2 \mathrm{D}$ rigid transformation, there corresponds exactly two unit DCN's with opposite signs.

Example 4. We compute the DCN's $\pm \hat{p} \in \check{\mathbb{C}}_{1}$ which represent $\theta$-rotation around $v \in$ $\mathbb{C}$. It is the composition of the following three rigid transformations: $(-v)$-translation, $\theta$-rotation around the origin, and $v$-translation. Thus, we have

$$
\hat{p}=\left(1+\frac{v}{2} \varepsilon\right) \cdot\left( \pm e^{\frac{\theta}{2} i}\right) \cdot\left(1-\frac{v}{2} \varepsilon\right)= \pm\left(e^{\frac{\theta}{2} i}+\left(e^{-\frac{\theta}{2} i}-e^{\frac{\theta}{2} i}\right) \frac{v}{2} \varepsilon\right) .
$$

\section{Relation to The DUAL QUATERnion NUMBERS}

The following homomorphism

$$
\check{\mathbb{C}} \ni p_{0}+p_{1} \varepsilon \mapsto p_{0}+p_{1} j \varepsilon \in \hat{\mathbb{H}}
$$

is compatible with the involution and the conjugation, and preserves the norm. Furthermore, if we identify $v=(x, y) \in \mathbb{R}^{2}$ with $1+(x j+y k) \varepsilon$, the above map commutes with the action. From this point of view, DCN is nothing but a sub-ring of DQN.

Note also that $\breve{\mathbb{C}}$ can be embedded in the ring of the $2 \times 2$-complex matrices by

$$
p_{0}+p_{1} \varepsilon \mapsto\left(\begin{array}{cc}
p_{0} & p_{1} \\
0 & \bar{p}_{0}
\end{array}\right) \text {. }
$$

Then

$$
\overline{\left(\begin{array}{cc}
p_{0} & p_{1} \\
0 & \bar{p}_{0}
\end{array}\right)}=\left(\begin{array}{cc}
\bar{p}_{0} & p_{1} \\
0 & p_{0}
\end{array}\right),\left|\left(\begin{array}{cc}
p_{0} & p_{1} \\
0 & \bar{p}_{0}
\end{array}\right)\right|^{2}=\operatorname{det}\left(\begin{array}{cc}
p_{0} & p_{1} \\
0 & \bar{p}_{0}
\end{array}\right) .
$$

We thus have various equivalent presentations of DCN. However, our presentation of $\check{\mathbb{C}}$ as the anti-commutative dual numbers is easier to implement and more efficient.

\section{INTERPOLATION OF 2D RIGID TRANSFORMATIONS}

First, recall that for the positive real numbers $x, y \in \mathbb{R}_{\geq 0}$, there are two typical interpolation methods:

$$
(1-t) x+t y, \quad t \in \mathbb{R}
$$

and

$$
\left(y x^{-1}\right)^{t} x, \quad t \in \mathbb{R} .
$$

The first method can be generalized to DQN as the Dual quaternion Linear Blending in [5]. Similarly, a DCN version of Dual number Linear Blending (DLB, for short) is given as follows: 
Definition 5. For $\hat{p}_{1}, \hat{p}_{2}, \ldots, \hat{p}_{n} \in \check{\mathbb{C}}_{1}$, we define

$$
\operatorname{DLB}\left(\hat{p}_{1}, \hat{p}_{2}, \ldots, \hat{p}_{n} ; w_{1}, w_{2}, \ldots, w_{n}\right)=\frac{w_{1} \hat{p}_{1}+w_{1} \hat{p}_{2}+\cdots+w_{n} \hat{p}_{n}}{\left|w_{1} \hat{p}_{1}+w_{1} \hat{p}_{2}+\cdots+w_{n} \hat{p}_{n}\right|},
$$

where $w_{1}, w_{2}, \ldots, w_{n} \in \mathbb{R}$. Note that the denominator can become 0 and so it is not always well-defined.

A significant feature of DLB is that it is distributive (it is called bi-invariance in some literatures). That is, the following holds:

$$
\hat{p}_{0} \operatorname{DLB}\left(\hat{p}_{1}, \hat{p}_{2}, \ldots, \hat{p}_{n} ; w_{1}, w_{2}, \ldots, w_{n}\right)=\operatorname{DLB}\left(\hat{p}_{0} \hat{p}_{1}, \hat{p}_{0} \hat{p}_{2}, \ldots, \hat{p}_{0} \hat{p}_{n} ; w_{1}, w_{2}, \ldots, w_{n}\right) .
$$

This property is particularly important when transformations are given in a certain hierarchy such as in the case of skinning; if the transformation assigned to the root joint is modified, the skin associated to lower nodes is deformed consistently.

Next, we consider interpolation of the second type. For this, we need the exponential and the logarithm maps for DCN.

Definition 6. For $\hat{p}=p_{0}+p_{1} \varepsilon \in \check{C}$, we define

$$
\exp \hat{p}=\sum_{n=0}^{\infty} \frac{\left(p_{0}+p_{1} \varepsilon\right)^{n}}{n !}=e^{p_{0}}+\frac{\left(e^{p_{0}}-e^{\bar{p}_{0}}\right)}{p_{0}-\bar{p}_{0}} p_{1} \varepsilon .
$$

When $\exp \hat{p} \in \check{\mathbb{C}}_{1}$, we can write $p_{0}=\theta i$ for some $\theta \in \mathbb{R}$, and

$$
\exp \hat{p}=e^{\theta i}+\frac{\sin \theta}{\theta} p_{1} \varepsilon
$$

For $\hat{q}=e^{\theta i}+q_{1} \varepsilon \in \check{\mathbb{C}}_{1}$, we define

$$
\log (\hat{q})=\theta i+\frac{\theta}{\sin \theta} q_{1} \varepsilon
$$

As usual, we have $\exp (\log (\hat{q}))=\hat{q}$ and $\log (\exp (\hat{p}))=\hat{p}$. Note that this gives the following Lie correspondence (see [1],[6])

$$
\begin{aligned}
& \exp : \mathfrak{d} \mathfrak{c} n \rightarrow \check{\mathbb{C}}_{1}, \\
& \log : \check{\mathbb{C}}_{1} \rightarrow \mathfrak{d} \mathfrak{c n},
\end{aligned}
$$

where $\mathfrak{d} \mathfrak{c} \mathfrak{n}=\left\{\theta i+p_{1} \varepsilon \in \check{\mathbb{C}} \mid \theta \in \mathbb{R}, p_{1} \in \mathbb{C}\right\} \simeq \mathbb{R}^{3}$. We have the following commutative diagram:

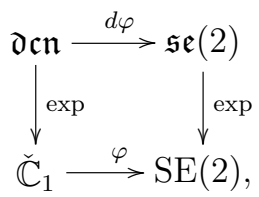

where

$$
\begin{aligned}
d \varphi: \mathfrak{d} \mathfrak{n} & \rightarrow \mathfrak{s e}(2) \\
\theta i+(x+y i) \varepsilon & \mapsto\left(\begin{array}{ccc}
0 & -2 \theta & 2 x \\
2 \theta & 0 & 2 y \\
0 & 0 & 0
\end{array}\right) .
\end{aligned}
$$

The following is a DCN version of SLERP [7]. 
Definition 7. SLERP interpolation from $\hat{p} \in \check{\mathbb{C}}_{1}$ to $\hat{q} \in \check{\mathbb{C}}_{1}$ is given by

$$
\operatorname{SLERP}(\hat{p}, \hat{q} ; t)=\left(\hat{q} \hat{p}^{-1}\right)^{t} \hat{p}=\exp \left(t \log \left(\hat{q} \hat{p}^{-1}\right)\right) \hat{p},
$$

where $t \in \mathbb{R}$.

\section{Application}

We developed the 2D probe-based deformer ([4]) for tablet devices running OpenGL ES (OpenGL for Embedded Systems). Thanks to the efficiency of DCN and touch interface, it offers interactive and intuitive operation.
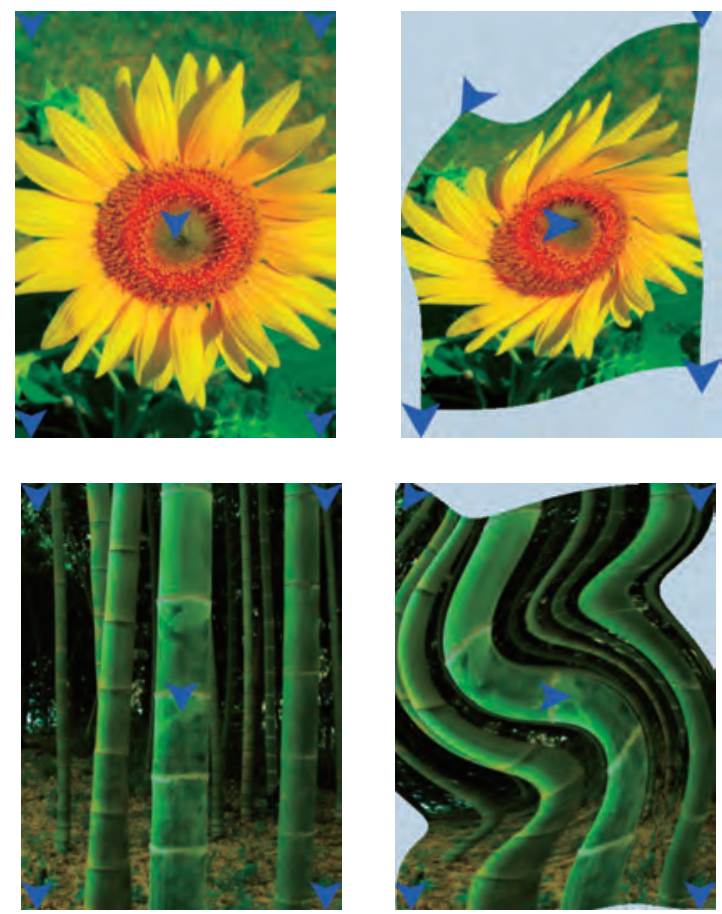

Figure 1. Left: set up initial positions of (blue) probes. Right: the target picture is deformed according to user's action on the probes.

\section{ACKNOWLEDGEMENTS}

This work was supported by Core Research for Evolutional Science and Technology (CREST) Program "Mathematics for Computer Graphics" of Japan Science and Technology Agency (JST). The authors are grateful for S. Hirose at OLM Digital Inc., and Y. Mizoguchi, S. Yokoyama, H. Hamada, and K. Matsushita at Kyushu University for their valuable comments.

\section{REFERENCES}

[1] J. E. Humphreys, Introduction to Lie Algebras and Representation Theory, Second printing, revised. Graduate Texts in Mathematics, 9. Springer-Verlag, New York, 1978.

[2] J. Jeffers, Lost Theorems of Geometry, The American Mathematical Monthly Vol. 107, No. 9, pp. 800-812, 2000. 
[3] S. Kaji, S. Hirose, H. Ochiai, and K. Anjyo, A concise parameterization of affine transformation, in preparation.

[4] S. Kaji, S. Hirose, H Ochiai, and K. Anjyo, A Lie theoretic parameterization of Affine transformation, in this volume.

[5] L. Kavan, S. Collins, J. Zara, C. O'Sullivan. Geometric Skinning with Approximate Dual Quaternion Blending. ACM Transaction on Graphics, 2008, 27(4), 105.

[6] H. Ochiai, Mathematical formulation of motion/deformation and its applications, in this volume.

[7] K. Shoemake, Animating rotation with quaternion curves, ACM SIGGRAPH, pp. 245-254, 1985. 